\title{
Conformational Dynamics of Heme Pocket in Myoglobin and Hemoglobin
}

\author{
Seongheun Kim, Jeonghee Heo, and Manho Lim
Deparment of Chemistry and Chemistry Institute for Functional Materials. Pusan National Lniversin, Busan 609-735, Korea
${ }^{*}$ E-mail: mhimiäpusan.ac.kr
Received Octoher 29.2004

\begin{abstract}
The conformational dy namics of heme pocket, a small vacant site near the binding site of heme proteins myoglobin $(\mathrm{Mb})$ and hemoglobin $(\mathrm{Hb})$. was investigated after photoly sis of carbon monoxide from $\mathrm{MbCO}$ and $\mathrm{HbCO}$ in $\mathrm{D}_{2} \mathrm{O}$ solution at $283 \mathrm{~K}$ by probing time-resolved vibrational spectra of photolyzed $\mathrm{CO}$. Two absorption bands. arising from $\mathrm{CO}$ in the heme pocket, evolve nonesponentially in time. The band at higher energy side blue shifts and broadens with time and the one at lower energy side narrows significantly with a negligible shift. These spectral evolutions are induced by protein conformational changes following photoly sis that modify structure and electric field of heme pocket. and ligand dynamics in it. The conformational changes affecting the spectrum of photolyzed $\mathrm{CO}$ in heme pocket likely modulates ligand-binding activity.
\end{abstract}

Key Words : Conformational dynamics. Myoglobin. Hemoglobin. Time-resolved IR spectroscopy. Heme pocket

\section{Introduction}

The conformation and dynamics of a protein can influence its activity. resulting in a structure-function-dynamics relationship: one of the most fundamental problems in biophysics. Heme proteins, nyoglobin $(\mathrm{Mb})$ and hemoglobin $(\mathrm{Hb})$. that reversibly binds small ligands such as $\mathrm{O}_{2}$. $\mathrm{CO}$. or NO, have long served as model systems for the experimental and theoretical investigation of protein structure and dynamics and its relation to function. ${ }^{1}$ Myoglobin is a monomeric protein that contains a heme prosthetic group [iron(II)-porphyrin group] and $\mathrm{Hb}$ is a tetrameric protein that consists of two $\alpha$ chains and two $\beta$ chains. each of which contains a single heme group. Several peptides in the vicinity of the heme are highly conserved in various manumalian species and $\alpha$ and $\beta$ chains of $\mathrm{Hb} .^{5}$ The highly conserved residues enconipasses heme pocket, a small vacant site near the active binding site. It has been suggested that the heme pocket serves as a station mediating the passage of ligands to and from the active binding site, thereby modulating ligand-binding activity ${ }^{6-8}$ To understand the role of the heme pocket and its mechanism how to mediate ligand transport, it is crucial to know detailed structural dynamics of the heme pocket.

$\mathrm{X}$-ray diffraction studies have shown that the tertiary structures of ligated and deligated heme proteins are different $^{3, p-1]}$ Thus ultrafast dissociation of $\mathrm{CO}$ from the heme-CO complex. optically triggerable. generates deligated heme proteins in the $\mathrm{CO}$-ligated conformation and initiates a change in the tertiary conformation of the protein that eventually leads to the quaternary conformational change in $\mathrm{Hb}$. The cooperative $\mathrm{O}=$ binding of $\mathrm{Hb}$ results from these conformational changes. The conformational relaxation in the vicinity of the heme can modulate the barrier to ligand binding and has been suggested to be responsible for the nonexponential geminate recombination. ${ }^{13-18}$ Conformational relaxation after photolysis of ligated heme proteins have been studied by numerous time-resolved spectroscopic methods probing Soret band absorption, ${ }^{16 \cdot 18}$ band III absorption, ${ }^{1+.17}$ pump-induced refractive index. ${ }^{19.211}$ and various vibrational bands for confonnation marker including $\mathrm{Fe}$-His stretching mode $\mathrm{e}^{\text {t1 }}$ and amide band ${ }^{2-24}$ These studies have measured various time scales for the conformational changes ranging from picoseconds to milliseconds due to differing sensitivity of the probe. There can be milliards of protein conformational motions and each experiment probes a fraction of the motion. Probing functionally important conformational motion is essential to understand protein dynamics and function relationship.

Probing structural changes influencing heme pocket is expected to be valuable because it plays a functionally important role in ligand binding and escape. However, direct probe of structural changes in heme pocket has been rarely realized due to lack of an appropriate probe for the structure in real time. Information on the structure near heme pocket has been indirectly inferred from spectral changes of the strongly absorbing heme. ${ }^{14.17}$ Recent time-resolved $x$-ray crystallography ${ }^{25.27}$ has observed all the structural evolutions of $\mathrm{Mb}$ after photolysis of $\mathrm{MbCO}$ at an atomic detail up to 150 ps time resolution ${ }^{27}$ The time-resolved electron density maps of $\mathrm{Mb}$ unveiled detailed structural changes of the protein including the heme pocket as it executes its function ${ }^{27}$ However, current time resolution of the $x$-ray study is not fast enough to follow the primary motion of heme proteins after photolysis. The tertiary conformation of $\mathrm{Mb}$ after photolysis of $\mathrm{MbCO}$ is known to evolve on picosecond time scale. ${ }^{1+17}$ It has been shown that majority of the primary motion occurs within $100 \mathrm{ps} .^{13.14}$ Even if timeresolved $\mathrm{x}$-ray crystallography can probe all the relevant time scale for protein motion. time-resolved spectroscopic investigations of a protein in physiologically relevant condition is useful to properly evaluate structural evolution 
observed by time-resolved $x$-ray crystallography ${ }^{27}$ because the $\mathrm{x}$-ray crystallography cannot assess thermally driven. functionally important conformational fluctuations occurring under physiologically relevant condition.

Vibrational spectrum of diatomic ligand is so sensitive to its environment that it can provides the means to probe the structural changes of its surroundings. Mid-IR absorption spectra of $\mathrm{CO}$ were measured $100 \mathrm{ps}$ after photodissociation from $\mathrm{MbCO}$ and $\mathrm{HbCO}{ }^{6}$ These spectra revealed two narrow vibrational features attributed to $\mathrm{CO}$ localized in rotationally constrained heme pocket. Two vibrational features were assigned to $\mathrm{CO}$ oriented oppositely in the same site within the heme pocket. ${ }^{6}$ Fentosecond polarization anisotropy midIR spectroscopy has shown that the photodissociated $\mathrm{CO}$ rotates and becomes trapped within a ligand-docking site located near the binding site. ${ }^{8}$ The spectra of photolyzed $\mathrm{CO}$ at $100 \mathrm{ps}$ are well described as a dipole bathed in a Stark field whose out-of-plane motion is constrained by a simple double-well potential. ${ }^{\text {ts }}$ The orientational distribution of photolyzed $\mathrm{CO}$ was determined experimentally ${ }^{\text {s }}$ The location of photolyzed $\mathrm{CO}$ in heme pocket was identified by correlating distribution of $\mathrm{CO}$ with two high probability regions found in a recent molecular dynamics (MD) sinulation. $^{2 s}$ These experimental results support the suggestion that the heme pocket mediates ligand transport to and from the active binding site and plays significant role in the function of ligand binding. Time-resolved spectra of photolyzed $\mathrm{CO}$ could evolve with ligand motion and structural changes of functionally important heme pocket induced by protein conformational relaxation after photolysis. While previous investigations of spectra for photolyzed $\mathrm{CO}$ have established functional significance of heme pocket as well as the orientation and dynamics of $\mathrm{CO}$ within the heme pocket. spectral evolution of $\mathrm{CO}$ in the heme pocket has not been studied systematically. ${ }^{6-8,28,239}$ The role of quaternary contacts to functionally important conformational changes can be obtained by comparing the dynamics of $\mathrm{Mb}$ and $\mathrm{Hb}$. Here we report conformational dynamics of heme pocket probed by following spectral evolution of photolyzed $\mathrm{CO}$ in heme pocket after photolyzing $\mathrm{CO}$ from $\mathrm{MbCO}$ and $\mathrm{HbCO}$. Kinetics of conformational change of heme pocket found to be similar to that of the iron-out-of plane displacement. indicating that ligand in the heme pocket is strongly influenced by the iron motion. We have also found that the spectral evolution of $\mathrm{CO}$ in $\mathrm{Mb}$ is similar to that in $\mathrm{Hb}$. implying that functionally important conformational change is similar in $\mathrm{Hb}$ and $\mathrm{Mb}$.

\section{Materials and Methods}

The details of the time-resolved mid-IR spectrometer was described elsewhere. ${ }^{3(1}$ Briefly, two identical home-built optical parametric aniplifiers (OPA). pumped by a commercial Ti:sapphire amplified pulse. are used to generate visible pump pulse ${ }^{\hat{\jmath} 1}$ and mid-IR probe pulse. ${ }^{31,3 \hat{3}}$ Pump pulse at 580 $\mathrm{num}$ with $3 \mu \mathrm{J}$ of energy was generated by frequency doubling of a signal pulse of one OPA in a type-I. 1-nm-thick
BBO crystal. ${ }^{31}$ Tunable mid-IR probe pulse with duration of 110 fs was generated by difference frequency mixing of the signal and idler pulse of the other OPA in a 1.5 -mm-thick, type-I AgGaS z crystal. After the sample is photolyzed by the optically delayed pump pulse, its transient mid-IR absorbance is measured with the probe pulse. The polarization of the pump pulse was set at the magic angle (54.7) relative to the probe pulse to recover isotropic absorption spectrum.

The broadband transmitted probe pulse is detected with a $\mathrm{N}_{2}(\mathrm{~L})$-cooled $\mathrm{HgCdTe}$ array detector which consists of 64 elements. The array detector is mounted on the focal plane of a $320 \mathrm{~mm}$ IR monochromator with a $150 \mathrm{lmm}$ grating. resulting in a spectral resolution of $c a .1 .4 \mathrm{~cm}^{-1} /$ pixel at $2100 \mathrm{~cm}^{-1}$. The signals from each of the detector elements were amplified with a home-built 64-channel amplifier and digitized by a 12-bit analog-to-digital converter. Chopping the pump pulse at half the repetition frequency of the laser and computing the difference between the pumped and unpumped absorbance determine the pump-induced change in the absorbance of the sample $\Delta A$. Due to the excellent short-term stability of the IR light source $(<1 \% \mathrm{mms})$, less than $1 \times 10^{-4} \mathrm{mms}$ in absorbance units after $0.5 \mathrm{sec}$ of signal averaging is routinely obtained without single shot referencing with an independent detector. The pump spot was made larger than the probe spot to ensure spatially uniform photoexcitation across the spatial dimensions of the probe pulse. ${ }^{2-4}$ Time resolution attained in this work. determined by transient absorption of $\mathrm{Si}$ wafer. is $<180 \mathrm{fs}$ full width at half maximum.

A $10 \mathrm{mM} \mathrm{Mb}{ }^{13} \mathrm{CO}$ was prepared by dissolving lyophilized skeletal horse $\mathrm{Mb}$ (Sigma) in deosygenated $\mathrm{D}_{2} \mathrm{O}$ (Merck, $99.9 \% \mathrm{D})$ buffered with $0.1 \mathrm{M}$ potassium phosphate $(\mathrm{pD}$ 7.4). The solution was equilibrated with $1 \mathrm{~atm}$ of ${ }^{13} \mathrm{CO}$ (Aldrich. $99.3 \%{ }^{13} \mathrm{C} .<1.8 \%,{ }^{18} \mathrm{O}$ ) and reduced with $c a .2$ equivalent concentration of freshly prepared sodium dithionate (Aldrich). The reduced $\mathrm{Mb}$ was stirred under $\mathrm{I}$ atm ${ }^{13} \mathrm{CO}$ for at least $20 \mathrm{~min}$. A $2.7 \mathrm{mM} \mathrm{Hb}{ }^{13} \mathrm{CO}$ (1l $\mathrm{mM}$ in heme) was prepared in the same way as the preparation of $\mathrm{Mb}^{13} \mathrm{CO}$ using lyophilized human $\mathrm{Hb}$ (Sigma). Sample preparation was carried out at ice bath to minimize thermal denature. To remove light scattering sources such as dust particles and denatured protein aggregates. samples were filtered through a $5-\mu \mathrm{m}$ membrane filter before loading in a gas-tight 50- $/ \mathrm{m}$-pathlength sample cell with $\mathrm{CaF}_{2}$ windows During data collection the sample cell was rotated sufficiently fast so that each photolyzing laser pulse illuminated a fresh volume of the sample. The temperature of the rotating sample cell was $283 \pm 1 \mathrm{~K}$. The integrity and concentration of sample was checked using UV-Vis and FT-IR spectroscopy (Bruker. Equinox 55). Because water is a strong IR absorber. the sample was prepared in $\mathrm{D}_{2} \mathrm{O}$ using ${ }^{13} \mathrm{CO}$ to isotopically shift interested spectrum into a region with greater IR transmission.

\section{Results and Discussion}

Time-resolved mid-IR absorption spectra of photolyzed 

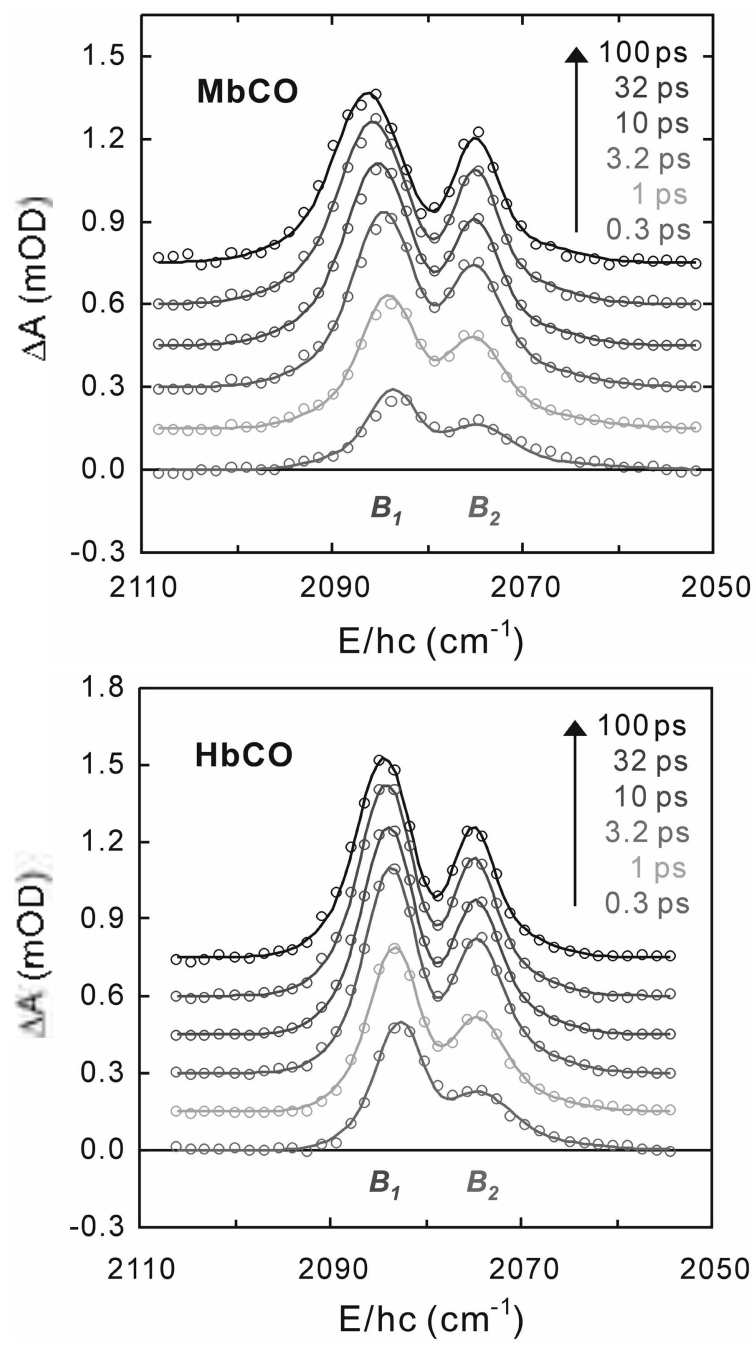

Figure 1. Representative time-resolved mid-IR absorbance spectrat of photolyzed ${ }^{1.3} \mathrm{CO}$ from $\mathrm{Mb}^{13} \mathrm{CO}$ and $\mathrm{Hb}^{1.3} \mathrm{CO}$ in $\mathrm{D}_{2} \mathrm{O}$ at $283 \mathrm{~K}$. The data (open circles) are fit to two evolving hands ( $B_{1}$ and $B_{2}$ ) and each band is modeled with a sum of two ciaussians (solid lines). For clarity, uxe cubic polynomial background and a small hot band have been subtracted from the measured spectra. Spectra have been offset one another to avoid overlap.

${ }^{13} \mathrm{CO}$ from $\mathrm{Mb}^{13} \mathrm{CO}$ and $\mathrm{Hb}^{13} \mathrm{CO}$ in $\mathrm{D}_{2} \mathrm{O}$ at $283 \mathrm{~K}$ were collected over the spectral range $2110-2050 \mathrm{~cm}^{-1}$ and a few of them at representative times are shown in Figure 1 . The two positive-going features (denoted $B_{1}$ and $\left.B_{2}\right)^{0.35}$ correspond to photodissociated ${ }^{1.9} \mathrm{CO}$ located in heme pocket with opposite orientation. ${ }^{6.828}$ ] wo features are apparent at $0.3 \mathrm{ps}$ (the earliest time shown) and fully developed in $<1 \mathrm{ps}$, consistent with ultrafast photodissociation of $\mathrm{CO}^{36.37}$ and the suggestion that $\mathrm{CO}$ is funneled into a heme pocket docking site near the binding site. ${ }^{8.28}$ Because escape of $\mathrm{CO}$ from the heme pocket and geminate rebinding of $\mathrm{CO}$ to $\mathrm{Mb}$ and $\mathrm{Hb}$ in aqueous solution at room temperature are much slower than $100 \mathrm{ps},{ }^{18}$ the time window of the present experiment, the magnitude of the features is expected to be maintained after an initial growth and that is the case. The initial growth of the features with a time constant of $1.1 \pm 0.1$ ps arises from protein rearrangement constraining the orientation of docked
$\mathrm{CO}$ after photolysis. ${ }^{8}$ Clearly, spectral evolution of $B$-states during the $100 \mathrm{ps}$ period results not from actual change of $\mathrm{CO}$ population in the heme pocket but structural changes at the heme pocket influencing $\mathrm{CO}$ dynamics.

Spectral evolution of $B$ bands is clearly visible throughout the pump-probe delay (see Figure 1). To characterize the spectral evolution, absorption features were modeled as a sum of Gaussian functions on a cubic polynomial background. "The cubic polynomial represents a small but broad absorption over the entire spectral range. which was also observed in previous experiments and was attributed to the effects of vibrational heating of the chromophore by the pump pulse. ${ }^{6.36 .39}$ Bectuse, while the probed spectral region is free from protein absorption. it is located in a tail of the strong O-D stretching mode of $\mathrm{D}_{2} \mathrm{O}$ at $2504 \mathrm{~cm}^{-1}\left(\xi_{\operatorname{mas}}=\right.$ $\left.71.5 \mathrm{M}^{-1} \mathrm{~cm}^{-1}\right){ }^{10}$ at slight change in $\mathrm{O}-\mathrm{D}$ absorption band by heating can cause a significant background shift in $\Delta 4$. The time dependency of the background signal was used to monitor the transfer of thermal energy from the chromophore to the solvent. The recovered time, $3-5 \mathrm{ps}$ is similar to that reported previously. ${ }^{39.11-1-1}$ Hot band, resulting from vibrationally excited $\mathrm{CO}$ (about $6 \%$ of the total population), was modeled as red-shifted replicas of $B$-states. ${ }^{6}$ The population of vibrationally excited $\mathrm{CO}$ was obtained from the fit of the integrated area of the hot band. The cubic polynomial background and the small hot band have been subtracted from the measured spectra for clarity. ${ }^{0.8}$

Previously $B_{1}$ has been modeled with a Gaussian and $B_{2}$ with a sum of two Gaussians. ${ }^{6}$ Two Gaussians for $B_{2}$ were not resolved and treated as if they were a single feature. ${ }^{6}$ The single feature consisting of a sum of two Gaussians depicts an asymmetric band, which likely arises from an anisotropic spatial distribution of photolyzed $\mathrm{CO}$ andior conformational substates of protein. ${ }^{6.28}$ The band position of $B$-state is mainly determined by the interaction between $\mathrm{CO}$ and the surrounding electric field. ${ }^{28}$ Both the spatial distribution of $\mathrm{CO}$ and the surrounding electric field can be anisotropic and modified by the structural inhomogeneity of protein, causing asymmetric band shape. With a better $\mathrm{S} N \mathrm{~N}$ ratio in the present experiment, we found that $B_{1}$ band also has an asymmetric shape and thus it was modeled with a sum of two Gaussians. In each band, the parameters for the small Gaussian were slaved to the main Gaussian by a shift for center wavenumber, a scale factor for standard deviation. and another scale factor for integrated absorbance. All these three parameters were set to the same throughout the whole spectra collected. Global fitting of the whole spectra using these three-shared parameters allowed us to recover robust spectral parameters characterizing $B$-states. An asymmetric band is better characterized by the moments than center wavenumber and standard deviation of each Gaussian. Here the first and the second moments, $M_{1}$ and $M_{2}$ are written by:

$$
M_{1}=\frac{\int \bar{v} \Delta A(\bar{v}) d \bar{v}}{\int \Delta A(\bar{v}) d \bar{v}}
$$




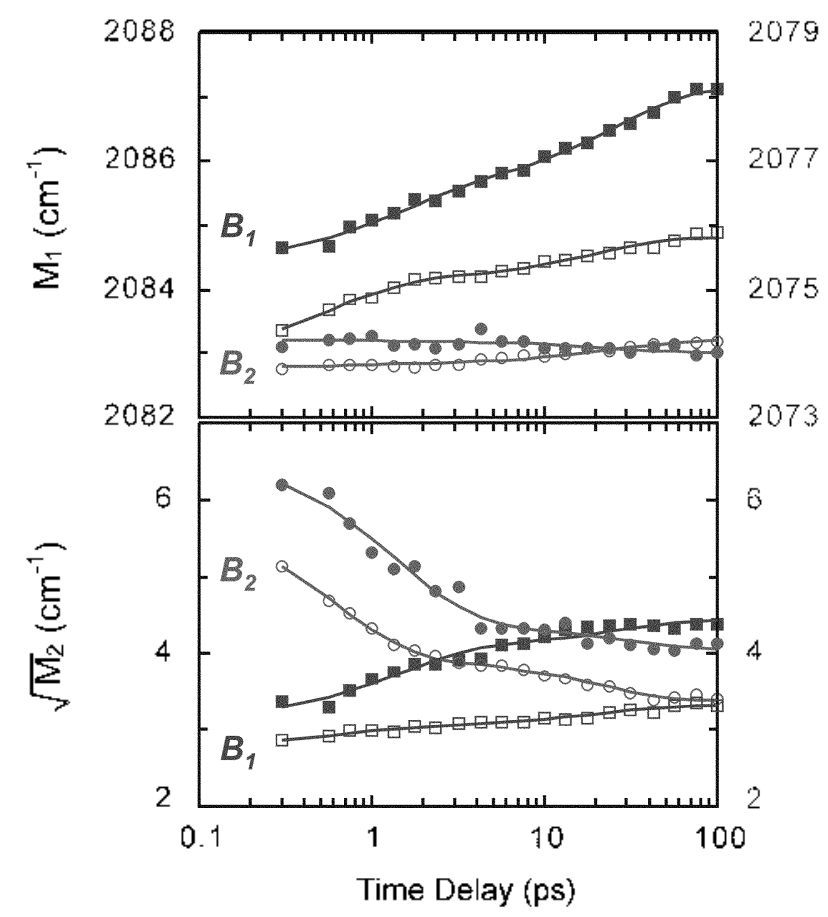

Figure 2, 'lime evolution of the moments describing bands $B_{1}$ (lelt ordinate) and $B_{2}$ (right ordinate) for photolyzed " $\mathrm{CO}$ from $\mathrm{Mb}^{13} \mathrm{CO}$ (filled symbols) and $\mathrm{Hb}^{13} \mathrm{CO}$ (open symbols) in $\mathrm{D}_{2} \mathrm{O}$. The moments, $M_{1}$ and $M_{2}$ are related to median navenumber and spectral sidth of the alssorption band. respectively. The data are well described by biexponential lunctions (solid lines).

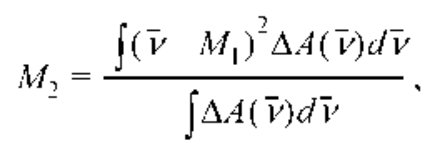

where $\bar{v}$ is the wavenumber in $\mathrm{cm}^{-1}$ unit. They correspond, respectively, to median wavenumber and variance of the asymmetric absorption band. The moments of each band are analytically calculated from the parameters of two Gaussians and plotted in Figure 2. While the spectral parameters for two Gaussians of each band had a slight scatter depending on the starting parameters for the fitting, the moments calculated from the Gaussian parameters were highly reproducible. The median wavenumbers and bandwidths of the $B$ states are comparable to those reported previously. $6.8 .28,35.36$

As can be seen in Figure 2, $B_{2}$ band narrows significantly at an essentially fixed or modestly shifting position, and $B_{1}$ band shifts toward higher energy and broadens. Spectral shift in $B_{1}$ for $\mathrm{MbCO}$ was the only observation reported previously and has been suggested to arise from conformational relaxation of the protein. ${ }^{8.29}$ Earlier studies may have lacked sensitivity needed to resolve change in bandwidth and modest $B_{2}$ band shift. Note that major change in bandwidth occurs within 5 ps the time range when data is on top of large solvent background. When the background is properly treated, even visual inspection of the spectra clearly reveals change in bandwidth and band position (see figure 1).

lime evolution for both moments of two bands was modeled by biexponential functions rate constants of which are shared for a given protein. Each moment is well reproduced by two amplitudes of biexponential function with the shared rate constants (solid lines in figure 2). Because the spectral evolution results from the same structural origin, it is reasonable to assume that the rates for each moment in a protein are the same. The recovered rate constants for $\mathrm{MbCO}$ are $(1.4 \mathrm{ps})^{-1}$ and $(26 \mathrm{ps})^{-1}$, and those for $\mathrm{HbCO}(0.6 \mathrm{ps})^{-1}$ and $(20 \mathrm{ps})^{-1}$. These rates are not intended to represent independent motion: they simply serve as parameters of evolving relaxation rates of the moments. Because protein motion occurs in broad range of time scale, ${ }^{, 5}$ it is physically more reasonable to consider evolving relaxation rates instead of a few discrete ones. However, the recovered rate constants are useful when comparing our results with earlier measurements on protein structural change reporting discrete rate constants. For example, in a MD simulation of $\mathrm{MbCO}$ photolysis ${ }^{13}$ a biexponential function was used to characterize the time-dependent ironheme displacement over a 100-ps period after ligand dissociation from $\mathrm{MbCO}$. The out-of-plane motion of the heme iron was suggested to be related to global $\mathrm{Mb}$ relaxation. Time constants from the simulation are 4.5-5 ps and 7.J-243 ps depending on the asymptotic value for the iron displacement. It is in reasonable agreement with our results. The evolution of the out-of plane iron position was also probed by monitoring evolution of band III, a weak Feporphyrin charge-transfer band near $763 \mathrm{~nm} .^{1.4}$ The evolution of band III position is highly nonexponential stretching over 5 decades in time. It was parameterized by a sum of three exponential functions with rate constants of $3.5,83$, and $3300 \mathrm{ps}$. A biexponential function can certainly describe the evolution of band III up to $100 \mathrm{ps}$ after photolysis of $\mathrm{MbCO}$ and the rate constants in this range is not far from our result. Clearly even if dynamics of highly nonexponential relaxation stretching many decades in time, it can be modeled with a biexponential function in a limited time range. For the shift of band III, when probed in broad range of time scale including fast timescale probing primary motion, a modified stretched exponential function was adequate to properly describe the nonexponential dynamics. ${ }^{\text {to }}$ Nonexponential conformational change of $\mathrm{Mb}$ was also explained by transition states of a single energy connecting two groups of protein conformational substates (the initial and final substates)..$^{16}$

While the overall spectral shift of $B_{1}$ in $\mathrm{Hb}$ is smaller than that of $\mathrm{Mb}$, the percent shift referenced to its own bandwidth is about the same, $-60 \%$ of $\left(\mathrm{M}_{2}\right)^{1 / 2}$ at 100 ps for both proteins. Because the conformational change of heme proteins continues up to milliseconds time scale ${ }^{18.47 .18}$ and the band position can be influenced by the conformational change, the spectral shift of $B_{1}$ is expected to continue beyond $100 \mathrm{ps}$. In contrast the bandwidth appears to approach to the asymptotic value by $100 \mathrm{ps}$. The frequencydependent anisotropy of $B$-state spectra was well described by a dipole bathed in a Stark field. ${ }^{\text {?8 }}$ In this description bandwidth of $B$-state spectrum results from orientational 


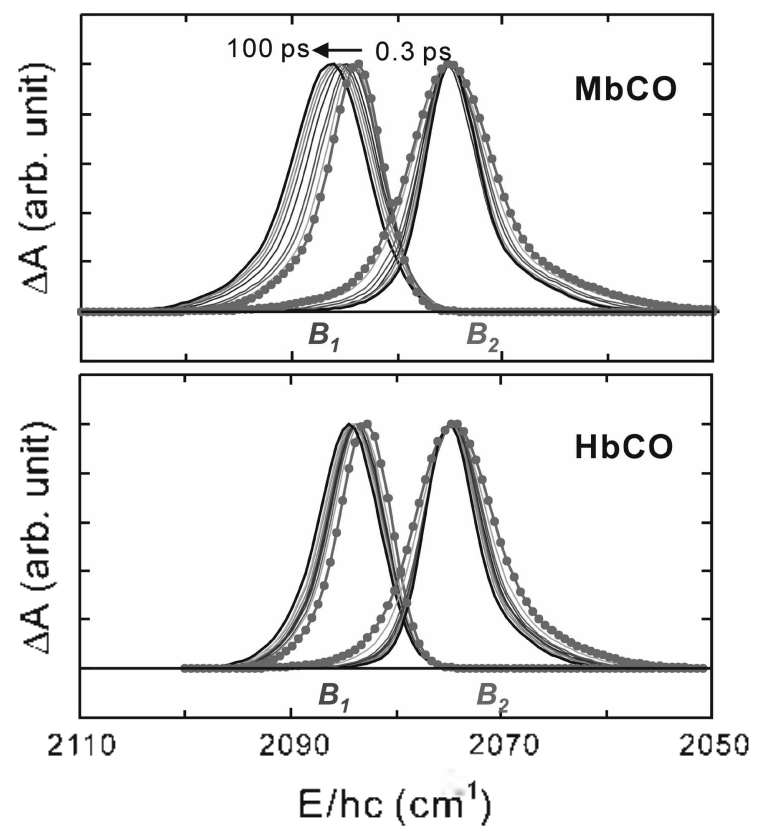

Figure 3. 'lime evolution of normalized hands for photolyed ${ }^{13} \mathrm{CO}$ Irom $\mathrm{Mb}^{1 .} \mathrm{CO}$ and $\mathrm{Ib}^{1 .} \mathrm{CO}$ in $\mathrm{D}_{2} \mathrm{O}$. The earliest spectra (at $0.3 \mathrm{ps}$ ) are shown $n$ ith the filled cireles on the thick solid lines. the latest spectra (at $100 \mathrm{ps}$ ) it ith the thick solid lines and the spectra at the intermediate times with thin solid lines.

distribution of photolyzed $\mathrm{CO}$ in the electric field at the heme pocket. Bandwidth is determined by distribution of $\mathrm{CO}$ and the magnitude of the electric field. If the electric field gets larger for a given distribution, the band position shifts and bandwidth broadens simultaneously (As the electric field increases, the shift of each $\mathrm{CO}$ increases for a given $\mathrm{CO}$ distribution and thus bandwidth broadens). If the distribution of $\mathrm{CO}$ varies for a fixed electric field. bandwidth varies without affecting band position. In this model, bandwidth is dominated by the interaction of distributed $\mathrm{CO}$ and the electric field; other inhomogeneous contribution to the bandwidth was found to be small. The percent evolution of bandwidths of $B_{1}$ and $B_{2}$ for $\mathrm{MbCO}$ are $\cdots 30 \%$ and $\cdots 60 \%$ of its own $\left(\mathrm{M}_{2}\right)^{1: 2}$ at $100 \mathrm{ps}$, respectively and those for $\mathrm{HbCO}$ are $-20 \%$ and $-70 \%$. Normalized spectra of each band are shown in Figure 3 to display the characteristics of spectral evolution more clearly. $B$ / band appears to broaden spectral shape by adding components having higher stretching mode. $B_{2}$ band simply narrows with time. Immediately after photodissociation of $\mathrm{CO}$ from $\mathrm{MbCO}$, structure surrounding heme pocket is not optimum for deoxy structure and thus it undergoes conformational relaxation. Naturally heme pocket geometry becomes more compact as protein conformation relaxes. In other words, as conformation of photolyzed $\mathrm{Mb}$ relaxes toward deoxy structure, heme pocket likely becomes more compact leading to narrower $\mathrm{CO}$ distribution, resulting in band narrowing.

A recent $\mathrm{MD}$ simulations revealed that photolyzed $\mathrm{CO}$ is constrained in an L-shaped thin slab containing two higher probability regions. ${ }^{40}$ It has been suggested that $B_{1}$ and $B_{2}$ correlate with the $\sim 125^{\circ}$ and $-90^{\circ}$ orientations found for the
I.3 $\AA$ and $2.3 \AA$ (from the heme normal) high probability sites in the MD simulations, respectively. ${ }^{28}$ According to the calculated electric field for a model heme-imidazole group using ab initio methods, there is a large change in the electric field as the iron moves relative to the herne plane and the $E$ field is the strongest near the iron. ${ }^{50}$ Various experiments and MI) simulations have shown that after photolysis of $\mathrm{MbCO}$ the iron moves out of the heme plane and its motion is governed by the global conformational relaxation. These experimental observations suggest that conformational relaxation varies electric field at heme pocket, which can induce spectral change of $B$-bands. Consequently spectral evolution of $B$-states is an excellent probe for protein conformational relaxation that affects ligand motion in heme pocket. In what follows we will assume that $B_{1}$ and $B_{2}$ correspond to two high probability sites: $B_{1}$ is assigned to the site $1.3 \AA$ apart and $125^{\circ}$ orientation from the heme normal and $B_{2}$ to the site $2.3 \AA$ apart and $90^{\circ}$ orientation. It is quite feasible that the electric field at $B_{1}$, nearer to the iron, varies more than that at $B_{2}$ as the iron moves out of the heme plane. Blue shifting and broadening of $B_{1}$ band indicate that the electric field at $B_{1}$ increases as conformational relaxation proceeds. Negligible shift and narrowing of $B_{2}$ band suggest that the electric field at $B_{2}$ is not much influenced by the iron motion and ligand at $B_{2}$ experiences tighter packing. When both $B_{1}$ and $B_{2}$ experience the packing of the heme pocket causing bandwidth narrowing, $B_{1}$ bandwidth can still increase if bandwidth broadening induced by the electric field is larger than bandwidth narrowing by the packing.

Rate constants for the relaxation of the moments for the $B$ band in Mb are, if not longer, similar to those in $\mathrm{Hb}$. While $\mathrm{Hb}$ has quaternary structure, the time scale for change of the quaternary structure is much longer than $100 \mathrm{ps}$, the present experimental time window. Quaternary contacts of $\mathrm{Hb}$ were suggested to retard conformational relaxation causing smaller blue shift for $B_{1}$ in $\mathrm{Hb}$ during $100 \mathrm{ps}{ }^{6}$ It may be responsible for the narrower bandwidth of $B$ state in spite of tetrameric structure with the $\alpha$ and $\beta$ chains. Similarity of $B$ bands and its spectral dynamics between $\mathrm{Mb}$ and $\mathrm{Hb}$ suggests that dynamics as well as structure of heme pocket is highly conserved.

A a $580-\mathrm{nm}$ photon energy $(49.3 \mathrm{kcal} / \mathrm{mol})$ is larger than dissociation energy of $\mathrm{Fe}-\mathrm{CO}(16.2 \mathrm{kcal} / \mathrm{mol})^{51}$ and the reorganization energy of the protein is $2.4 \mathrm{kcal} / \mathrm{mol}^{.52}$ \% $T$ herefore, $30.7 \mathrm{kcal} / \mathrm{mol}$ of excess energy would be deposited into the heme group and departing $\mathrm{CO}$. Energy deposited to the heme is known to decay with 6 ps time scale by transferring energy to surrounding solvent via protein. ${ }^{42}$ Because the vibrationally excited $\mathrm{CO}$, appearing at the hot band. is less than $6 \%$ of total photolyzed $\mathrm{CO}$ population. most of $\mathrm{CO}$ is generated in the vibrational ground state. Evidently all the excess energy of the majority of departing $\mathrm{CO}$ is in rotational and translational degrees of freedom. These excess energies are transferred to the residues of the heme pocket by the first few collisions of the ligand after photodissociation $(<0.3$ ps). ". Thus in our experimental timescale (longer than 0.5 ps), most of $\mathrm{CO}$ is thermalized and its motion is diffusive. 
Consequently the dynanics of $\mathrm{CO}$ probed here after photol$\mathrm{y}$ sis is comparable to that of thermal $\mathrm{CO}$ in physiological condition. $^{8}$

In conclusion, we have measured the dynanics of conformational change affecting heme pocket of $\mathrm{Mb}$ and $\mathrm{Hb}$ after photolysis of CO-ligated heme proteins by probing spectral evolution of $B$ bands using femtosecond IR absorption spectroscopy. Position of $B_{1}$ band blue shifts and its width broadens nonexponentially, which is attributed to the variation of the electric field at $B_{1}$ induced by protein conformational relaxation after photolysis. Position of $B$ : band undergoes a negligible shift and its width narrows significantly. It is ascribed to tightening of the heme pocket arising from conformational relaxation. Spectral evolution of $B$ bands can serve as an excellent probe for conformational relaxation affecting ligand in heme pocket. an intermediate for the ligand binding reaction. This conformational relaxation likely plays a significant role in controlling the ligand-binding affinity.

Acknowledgement. This work was supported by grants from Korea Research Foundation Grant (KRF-2001-015DP0228). This research used femtosecond laser system in the Central Laboratory at Pusan National University.

\section{References}

1. Springer. B. A.: Sligar. S. G.: Olson. J. S.: Phillips. G. N.. Jr. Chem. Rev 1994. 94.699.

2. Perutz. M. F.: Mathews. F. S. J. Mol. Biol. 1966. 21. 199

3. Takano. T. J. Mol Biol 1977, 110. 569.

4. Stryer. L. Biochemishy; W. H. Freeman and Co.: San Francisco. 1988: Vol. 3rd.

5. Swiss-Prot Protein Datatase: National Center of Biotechnology Intormation: Bethesda. 1994.

6. Lim, M.: Tackson. T. A.: Anfintud, P. A. J. Chem. Phys. 1995. 102,4355 .

7. Lim. M.: Jackson. T. A.: Anfinrud, P. A. Science 1995. 269,962.

8. Lim. M.: Jackson. T. A.: Antinnud. P. A. Nature Sruct. Biot. 1997. 4. 209 .

9. Luisi. B.: Liddington. B.: Fermi. G.: Shibayama. N. J. Mol. Biol. $1990.214,7$.

10. Phillips. S. E. V. J. Mol Biol 1980. $1+2,531$.

11. Shaanan, B. J. Mol. Biol. 1983. 171.31.

12. Agmon. N.: Hoptield. J. J. J. Chemt. Phvs. 1983. 79. 2042.

13. Kuczera. K.: Lambry. J. C.: Martin. J. L.: Karplus. M. Proc. Natl. Acad Sci US.A. 1993. 90.5805.

14. Lim. M.: Jackson. T. A.: Anfinrud. P. A. Proc. Natl Acad Sci. L.S.A. 1993. 90.5801.

15. Petrich. J. W: Lambry I. C.: Kuczera. K.: Karplus. M: Poyart. C.: Martin. J. L. Biochemistry 1991. 30.3975.

16. Hagen. S. J.: Hotrichter. J.: Eaton. W. A. J. Phws. Chem. 1996. 100. 12008

17. Steinbach. P. I.: Ansari. A: Berendzen. I.: Braunstein. D.: Chu. K.: Cowen, B. R.: Ehrenstein, D.: Frauenfelder. H: Johnson, I. B. et al. Biochemistry 1991,30.3988.

18. Tian. W. D: Sage. J. T.: Srajer. V.: Champion. P. M. Phys. Rev Lett. 1992, 68, 408

19. Deak. J.: Chiu. H.: Lewis. C. M.: Miller. R. J. D. J. Phns. Chem. $B$ $1998,102,6621$
20. Genberg. L.: Richard. L.: McLendon. G.: Miller. R. T. D. Science 1991. 251. 1051 .

21. Findsen. E. W: Scott. T. W: Chance. M. R.: Friedman. J. M: Ondrias, M. R. J. Am. Chem. Soc. 1985. 107. 3355.

22. Causgrove, T. P.: Dver, R. B. Biochemistry 1993. 32, 11985

23. Causgrove, T. P.: Dver, R. B. J. Phus. Chem. 1996, 100. 3273

24. Kiml. S. Jitl. G.: Lim. M. Bull. Korean Chem. Soc. 2003. 24. 1470

25. Brunori. M.: Vallone. B.: Cutruzzola. F.: Travaglini-Allocatelli. C.: Berendzen. J.: Chu. K.: Sweet. R. M.: Schlichting. I. Proc. Katl Acad Sci. U.S.A. 2000, 97. 2058.

26. Chu. K.: Vojtchovsky, J: McMahon, B. H.; Sweet, R. M.: Berendzen, J.: Schlichting, I. Nature 2000, 403.921.

27. Schott. F.: Lim. M.: Tackson. T. A.: Smirnov. A.: Soman. J.: Olsont. I. S.: George N.: Phillips. T.: Wulffi. M.: Anfintud. P. A. Science 2003. 300. 1944

28. Lim. M.; Jackson. T. A.; Anfinrud. P. A. J. Am. Chem. Soc. 2004. 126.7946

29. Anfinrud, P. A.; Lim, M.: Jackson. T. A. Proc. SPIE-Int. Soc. Opt. Eng. (Longer Wavelengh Lasers and Applicationst 1994. 2138. 107.

30. Kiml. S.: Tin. G.: Lim. M. J. Phw Chem. B 2005. ASAP

31. Lim. M.: Wolford. M. F.: Hamm. P.: Hochstrasser, R. M. Chem. Phus. Lett. 1998. 290. 355

32. Hamm. P. Lim, L.: Hochstrasser, R. M. J. Phys. Chem. B 1998. 102.6123.

33. Hamm. P.: Kaindl. R. A.: Stenger. J. Opt. Lett. 2000. 25. 1798.

34. Lim. M. Bull. Korean Chem. Soc. 2002. 23. 865

35. Alben, J. O.: Beece, D.; Bowne. S. F.: Doster, W.: Eisenstein, L. Frauenfelder H.; Good, D.: MeDonald. J. D.; Marden, M. C.: Mo. P. P.: Reinisch. L.: Reynolds. A. H.: Shyamsunder. E.: Yue. K. T. Proc. Natl Acad Sci. LS. A. 1982. 79. 3744.

36. Antinirud. P. A.: Han1. C.: Hochstrasser. R. M. Proc. Natl Acad Sci. LS.A. 1989.86 .8387

37. Petrich. J. W. Povart. C.; Martin. J. L. Biochemisny 1988, 27 4049.

38. Henry, E. R.: Sommer, J. H:- Hofrichter, J.: Eaton. W. A. J. M fol. Biol. 1983. 166. 443 .

39. Lian1. T.: Locke. B.: Kholodenko. Y.: Hochstrasser. R. M. J. Phws Chent 1994. 98. 11648

40. Venvaminov, S. Y: Prendergast. F. G. Anat Biochem 1997. 248. 234

41. Li. P. Champion. P. M. Biophys. d. 1994. 66, 430

42. Lim. M.: Tackson. T. A.: Anfinrud. P. A. J. Phys. Chem. 1996. 100.12043

43. Lingle. R.. Ir: Xu. X.: Zhu. H.: Yu. S. C.: Hopkins. J. B. J. Phus Chem 1991, 95,9320.

4. Lingle, R., Jr: Xu. X.: Zhu. H.: Yu. S. C: Hopkins, J. B.; Straub. K. D. J Am. Chem Soc, $1991,113,3992$.

45. Brooks. C. L.. III: Karplus. M.: Pettitt. B. M. Proteins: a Theoretical Perspective of Ehmanics. Structure and Thermodnantics. John Wiley \& Sonts: New York. 1988.

46. Jackson, T. A.: Lim, M: Anfinrud, P. A. Chem. Phus. 1994, 180. 131

47. Ansari, A.; Jones, C. M.: Henry, E. R.: Hofrichter. J.: Eaton, W. A Biochentisty 1994. 33. 5128.

48. Murray. L. P.: Hofichter. J.: Henry. E. R.: Eaton. W. A. Biophn Chent 1988. 29.63

49. Meuwly, M: Becker, O. M.: Stote. R.: Karplus, M. Biophys. Chen 2002, 98,183

50. MoMahon. M. T; deDios. A. C.: Godtout, N.; Salzmann, R: Laws. D. D.: Le. H.: Haylin. R. H.: Oldfield. E. J. Ant. Chem. Soc. 1998. 120.4784.

51. Rudolph. S. A.: Boyle. S. O.: Dresden. C. F.: Gill. S. J Biochemisty 1972, 11. 1098

52. Srajer. V.: Champion. P. M. Biochemistry 1991. 30.7390. 